\title{
Optimal Consensus Set for Annulus Fitting
}

\author{
Rita Zrour, Gaëlle Largeteau-Skapin, and Eric Andres \\ Laboratory XLIM, SIC Department, \\ University of Poitiers BP 30179, UMR CNRS 6712 \\ 86962 Futuroscope Chasseneuil Cedex, France
}

\begin{abstract}
An annulus is defined as a set of points contained between two circles. This paper presents a method for fitting an annulus to a given set of points in a $2 \mathrm{D}$ images in the presence of noise by maximizing the number of inliers, namely the consensus set, while fixing the thickness. We present a deterministic algorithm that searches the optimal solution(s) within a time complexity of $O\left(N^{4}\right), N$ being the number of points.
\end{abstract}

Keywords: Digital geometry; shape fitting; consensus set; outliers; digital arc; annulus.

\section{Introduction}

Detection of basic geometric properties such as lines, planes and circles are essential tasks in the field of image analysis and computer vision. The main objective of this paper is annulus fitting to a given set of points. For instance, annulus fitting is useful for shape approximation [4] and image segmentation [6]. In this paper, we present a novel method that, given an arbitrary $2 \mathrm{D}$ point cloud, finds annuli of a given width that minimizes the number of outliers, or alternatively maximizes the number of inliers. One of the possible application of our method in the digital space is the fitting of discrete analytical circle of Andres [2]. The set of points which do fit the model is also called consensus set. The idea of using such consensus sets was proposed for the RANdom Sample Consensus (RANSAC) method [9, which is one of the most widely used in the field of computer vision. However RANSAC is inherently probabilistic in its approach and does not guarantee any optimality while our method is both deterministic and optimal in the size of the consensus set.

Different algorithms detecting annuli have been proposed. Most of these algorithms minimize the thickness of the annuli (digital circle). Among them, some consider that no noise is present in the image, and concentrate only on the problem of recognition instead of the fitting problem [16 51]. Some algorithms deal with outliers [7]10 but the number of outliers is usually predefined 10,11 and the problem consists in minimizing the width.

One frequently used approach in annulus recognition stems from the O'Rourke et al. [13] disk recognition method that transformed it into a problem of circular separability; they use a mapping that raises every point $(x, y)$ to the paraboloid

I. Debled-Rennesson et al. (Eds.): DGCI 2011, LNCS 6607, pp. 358-368, 2011.

(C) Springer-Verlag Berlin Heidelberg 2011 
$z=x^{2}+y^{2}$. Using this mapping, every circle in the primal space corresponds to a plane cutting the paraboloid in the dual space and thus circular separability is transformed to a plane separability in the dual space. This separability problem is then solved in linear time using Megiddo's or Dyer linear algorithms 812]. Recently, in the discrete geometry community, a simple online linear-time algorithm for recognition of digital circular arcs has been proposed in [15] based on the idea of O'Rourke et al. [13; this algorithm can be used in an incremental way, with a complexity $O\left(n^{4 / 3}\right)$ whenever a new point is added by using an optimization proposed in 3. 3. Using O'Rourke's mapping, an annulus of fixed area in the primal space, corresponds to two parallel planes of fixed vertical thickness in the dual space. The vertical thickness of the two parallel planes corresponding to an annulus is however not fixed when the thickness $w$ of the annuli and not the area of the annuli is fixed, as is the case of our fitting problem. In this case, the dual approach does not seem to simplify the problem.

To our knowledge there is no work neither in the continuous domain nor in the discrete domain that treated the problem of optimal annulus fitting with fixed width. Our main contribution is that we find exact solution(s) by minimizing the number of outliers.

The rest of the paper is organized as follows: in section 2 we expose the problem of annulus fitting, present some properties of annuli and explain how we can find all the consensus sets. In sections 3 we show how to build an annulus from three points. Section 4 provides the algorithm for finding the optimal annulus and shows some results. Finally Section 5 states some conclusions and perspectives.

\section{Annulus Fitting}

An annulus $A$ of width $\omega$ and radius $R$ centered at $C\left(C_{x}, C_{y}\right)$, is defined by the set of points in $R^{2}$ satisfying two inequalities:

$$
S=\left\{\left(P_{x}, P_{y}\right) \in \mathbb{R}^{2}: R^{2} \leq\left(P_{x}-C_{x}\right)^{2}+\left(P_{y}-C_{y}\right)^{2} \leq(R+\omega)^{2}\right\}
$$

where $C\left(C_{x}, C_{y}\right) \in \mathbb{R}^{2}$ and $R, \omega \in \mathbb{R}_{+}$.

Using the above annulus model, our fitting problem is then described as follows: given a finite set $S=\left\{\left(P_{x}, P_{y}\right) \in \mathbb{R}^{2}\right\}$ of $n$ points such that $n \geq 3$, and given a width $\omega$ we would like to find an annulus $A$ of width $\omega$ such that it contains the maximum number of points in $S$. Points $\left(P_{x}, P_{y}\right) \in S \cap A$ are called inliers; otherwise they are called outliers. It should be noted that $n \geq 3$ since if $S$ contains less than 3 points, the fitting problem has an infinity number of solutions.

We denote $B_{i}$ (resp. $B_{e}$ ) that we call the internal (resp. external) border of the annulus, the set of points located at distance $R$ (resp. $R+\omega$ ) from $C$.

\subsection{Annuli and Their Consensus Sets}

Our approach is focused on inlier sets, also called consensus sets. Since $S$ is finite, it is obvious that the number of different consensus sets for the annulus 
is finite as well. Thus, if we can find all different consensus sets $\mathcal{C}$ from a given set $S$, we just need to verify the size of each $\mathcal{C}$ and find the maximum one as the optimal solution. Then the following question comes up naturally: is it possible, given a width $\omega$ to find all the consensus sets of $S$ ? if the answer is positive, how can we do it? In the section 2.2, we will answer both questions by giving some properties related to annuli.

\subsection{Annular Characterizations}

The following theorem states that given a width $\omega$, and given an annulus $A$ covering a set of points $S$, there exists at least another annulus $A^{\prime}$ of same width, that covers $S$ and passes through at least 3 points of $S$.

Theorem 1. Let $S$ be a set of $n(n \geq 3)$ points in $\mathbb{R}^{2}$. Let $A=\left(C\left(C_{x}, C_{y}\right), R, \omega\right)$ be the annulus of center $C\left(C_{x}, C_{y}\right)$, of internal radius $R$ and of width $\omega$ such that $\forall\left(P_{x}, P_{y}\right) \in S, R^{2} \leq\left(P_{x}-C_{x}\right)^{2}+\left(P_{y}-C_{y}\right)^{2} \leq(R+\omega)^{2}$. Then it exists $A^{\prime}=\left(C^{\prime}\left(C_{x}^{\prime}, C_{y}^{\prime}\right), R^{\prime}, \omega\right)$ such that:

$$
\exists P_{0}, P_{1}, P_{2} \in S, \forall i \in[0,2], P_{i} \in B_{i} \text { or } P_{i} \in B_{e}
$$

Proof. Let $S$ be a set of $n(n \geq 3)$ points in $\mathbb{R}^{2}$. Let $A=\left(C\left(C_{x}, C_{y}\right), R, \omega\right)$ be the annulus of center $C\left(C_{x}, C_{y}\right)$ with internal radius $R$ and width $\omega$ such that $A$ covers $S$ : i.e. $\forall\left(P_{x}, P_{y}\right) \in S, R^{2}<\left(P_{x}-C_{x}\right)^{2}+\left(P_{y}-C_{y}\right)<(R+\omega)^{2}$. Our first assumption is that no point of $S$ is on the annulus borders.

The theorem proof is given in three steps: First we show that we can always decrease the radius so as to put at least one point of $S$ on the annulus external borders. We note this point $P_{0}$. In the second step, we show that a rotation centered on $P_{0}$ allows to put another point $P_{1}$ of $S$ on one border. The last step is more delicate since it consists in changing both the radius and the center position so that a third point $P_{2}$ is now on one of the borders of the annulus. Of course, if in any of these steps we put more than one point on the borders than we just skip one or more steps.

A First step: the radius decreasing. Let $R_{0}$ be the distance between the farthest point $P_{0}$ of $S$ from the center $C$ of $A$. The annulus $A_{0}=\left(C\left(C_{x}, C_{y}\right),\left(R_{0}-\right.\right.$ $\omega), \omega)$ is such that $P_{0}$ is on its external border $B_{e}$.

B Second step: the rotation centered on $P_{0}$. Since the rotation center is on the external border (see $[\mathrm{A}]$ ), we can continuously rotate $A_{0}$ to reach a second point $P_{1}$ of $S$.

We note $P_{1}$ the first point reached. Let $\operatorname{Rot}_{P_{0}, \theta}$ the rotation centered on $P_{0}$ with angle $\theta$, such that $\theta$ is the smallest angle verifying:

$$
\exists \theta \in[0,2 \pi], \exists P_{1} \in S, R^{2} \leq\left(P_{1_{x}}-C_{x}\right)^{2}+\left(P_{1_{y}}-C_{y}\right)^{2} \leq(R+\omega)^{2}
$$

We denote $A_{1}=\left(\operatorname{Rot}_{P_{0}, \theta}(C), R, \omega\right)$. 
C Third step: the radius variation. After the first two steps, we obtain an annulus $A_{1}$ that has two points $P_{0}$ and $P_{1}$ of $S$ on its borders. Two configurations can appear: either both points are on the border $B_{e}$, or the points are each on a different border (case iii).

i. Both points $P_{0}$ and $P_{1}$ are on $B_{e}$. In this case, any modification on the radius $R$ involves a move of the center along the bisector of segment $P_{0}$ and $P_{1}$. When the center $C$ passes exactly between both points, some problems may occur and we have to separate two cases:

$* d\left(P_{0} P_{1}\right) \geq 2 \omega$ : in this case, the center can pass between the points and there always exists an annulus of width $\omega$ passing through both points. All the points of $S$ can be reached this way and we necessarily encounter a third point of $S$. In Fig. 1 1 , we can see the annulus with the two extreme cases, when the radius is ${ }_{-}^{+} \infty$. Fig. 1 $\mathrm{b}$, shows an initial set of points with an initial annulus of center $C$ passing by two external border points $P_{0}$ and $P_{1}$ (colored black), and the rotated annulus (colored red) by moving the center toward $-\infty$ from $C$ to $C_{2}$ in order to reach a third points $P_{2}$. Fig. 1t shows the case when the center moves toward $+\infty$ in order to reach a third points $P_{2}$. The movement of the center between ${ }_{-}^{+} \infty$ allows to reach a third point.

* $d\left(P_{0} P_{1}\right)<2 \omega$ : when the points $P_{0}, P_{1}$ are on $B_{e}$, the circle having the two points on $B_{e}$ must have at least a radius of $\omega$ since if the radius is less than $\omega$, no internal circle and so no annulus can be built. However when $d\left(P_{0} P_{1}\right)<2 \omega$, the movement of the center from $C$ to $C_{2}$ to reach a third points $P_{3}$ cannot be done in all the cases. For example in Fig. 1 d d $d\left(P_{0} P_{1}\right)=\omega$ and in this case, the center cannot move along the green line since $\frac{\omega^{2}}{4}+b^{2}>=\omega^{2}$, i.e. $b$ must be greater than $\frac{\sqrt{3} \omega}{2}$. Therefore there exist some points of $S$ that cannot be reached. Fig. 1e shows a case where no annulus having three border points can be constructed from the annulus having $P_{0}$ and $P_{1}$ on $B_{e}$. When this is the case, we have to change the configuration: we choose as point $P_{2}$ the point closest to the limit and we build the annulus of width $\omega$ with internal circle passing through $P_{0}, P_{1}$ and $P_{2}$ (Fig. 1f).

ii. Both points are on different borders.

In Fig. 2a, b, c, there are three possible configurations of two points on different border of the annulus $A$. In each of these configurations, modifying the radius allows to reach almost all the initial set. If a point $P_{2}$ of $S$ is inside the green circle Fig. $2 \mathrm{~d}$, e , inside the annulus, it has to be reached by one of the annulus border. However, there exists an area that is not reached by such a variation (in dark in Fig. 2d, e).

If the points of $S$ are all in this dark part, we have to change our strategy: we choose as point $P_{2}$ of $S$ the closest to one of the extreme lines and we build the annulus of width $\omega$ such that the three points $P_{0}, P_{1}$ and $P_{2}$ are on $B_{e}$ (see Fig. $2 \mathrm{~b}, \mathrm{~h}$ ). This annulus thus covers the whole dark area where all the other points of $S$ are and it passes through 3 points of $S$. 

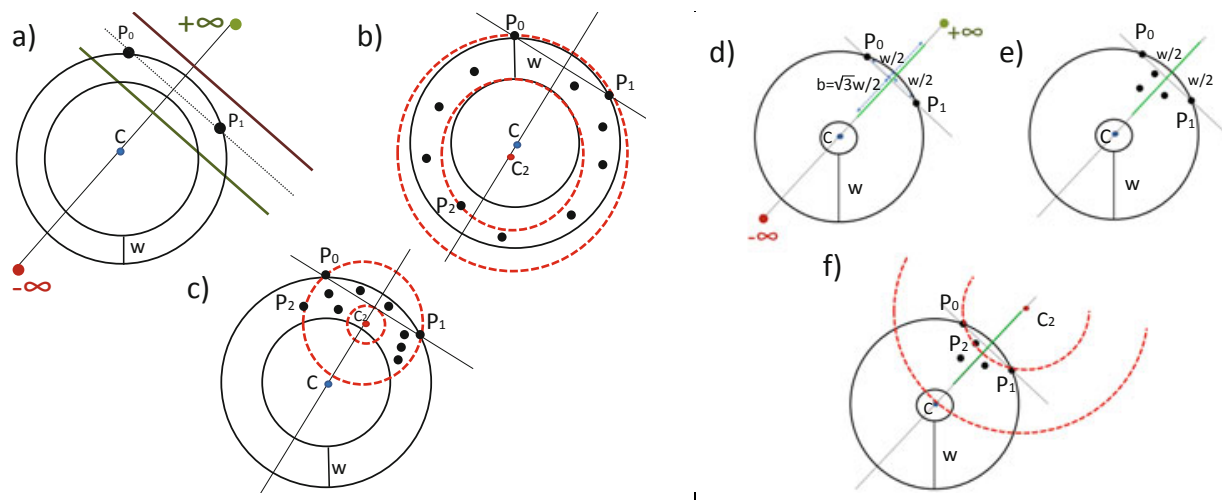

Fig. 1. Third step: a), b) and c), shows case ii where both points $P_{0}$ and $P_{1}$ are on the external border of $A$ and the distance is greater than $2 \omega$. The center $C$ colored blue must be moved along the line $-\infty$ in b) and $+\infty$ in c) in order to reach a third point $P_{2}$. The new center is $C_{2}$ and is colored in red, d), e), f) shows case ii where both points $P_{0}$ and $P_{1}$ are on the external border of $A$ and the distance between them is less than $2 \omega$. The configuration must be changed by choosing a point $P_{2}$ closest to the limit and the new annulus is the one colored in red.

In Fig. 2r, f, where both points are at distance $\omega$; we have to perform a rotation centered on one of both points until reaching another point $P^{\prime}$ on the border. If we rotate using $P_{0}$, the point $P_{1}$ in Fig. 2f no longer belongs to the border of the annulus. In this case the configuration can be viewed as the same configurations we already addressed: either $P^{\prime}$ and $P_{0}$ are on the internal border or $P^{\prime}$ is on the external border but not at the distance $\omega$ from $P_{0}$ (otherwise it would have been reached by a circle centered on $P_{0}$ with radius $\omega$ passing through $P_{1}$ and thus we would already have found the annulus we were searching for). Both cases leads to a third point.

In all cases, if an annulus of width $\omega$ covers $S$, then it is possible to build an annulus of same width that passes through 3 points of $S$.

\section{Building an Annulus of Width $\omega$ from Three Points}

The following theorem states that we can build a finite number of annuli of width $\omega$ from 3 points (see Fig. 3).

Theorem 2. There are at most 8 annuli of a given width $\omega$ passing through 3 given points $P_{1}, P_{2}$ and $P_{3}$ of $S$.

Proof.

- If the 3 points are on the same circle: depending on the radius of the circle one or two annuli can be build. If the radius of the circle is greater than $\omega$ 
a)

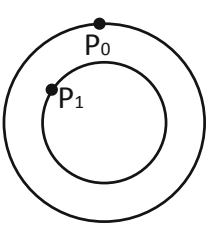

b)

c)
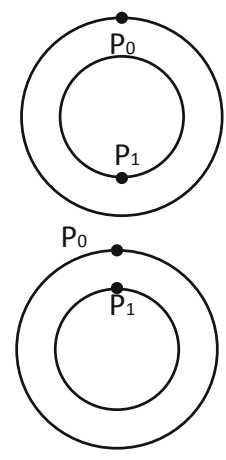

d)

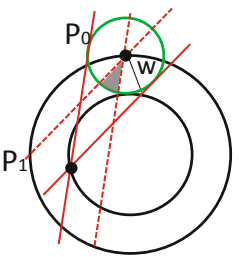

e)

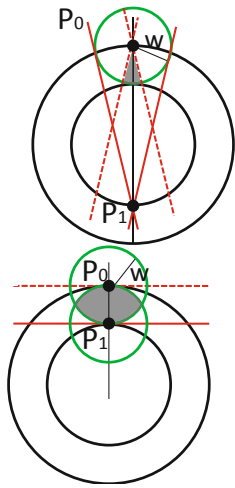

g)

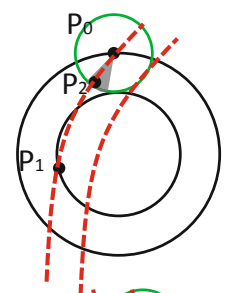

h)

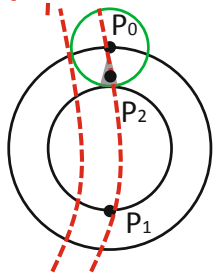

Fig. 2. Third step: case iii, both $P_{0}$ and $P_{1}$ are on different border of $A$

then 2 annular are built, the first having the points on the internal border and the other having the points on its external border. When the radius is less than $\omega$ then only one annulus having the three points on its internal border can be built.

- If 2 of the 3 points are on one border and the third one on the other: three possible configurations exists either $\left(P_{1}, P_{2}\right.$ or $P_{3}$ is alone on one border $)$ and for each of them we can buid at most two annuli (the lonely point is either on $B_{i}$ or on $B_{e}$ ). The principle of the building process is to build the two circles $C^{\prime}$ and $C^{\prime \prime}$ that passes through the 2 points on the same border and tangent (from the outside or inside) to the circle $c$ of radius $\omega$ centered on the third point. (see [14] for more details about the construction).

Fig. 3 shows the different cases of building the annulus, we assume that $P_{0}$ and $P_{2}$ are on one border and $P_{1}$ on the other. Fig. 33 shows the construction method; the circle $C$ of radius $\omega$ centered on $P_{2}$ is drawn (blue circle), then the two circles $C^{\prime}$ and $C^{\prime \prime}$ passing through $P_{0}$ and $P_{2}$ and tangent to $C$ are drawn (red circles). Fig. 3 b presents the particular case where $P_{0}$ is exactly at distance $\omega$ from $P_{1}$ (i.e. on the circle of center $P_{1}$ and radius $\omega)$. In this case there is a unique circle that passes through $P_{0}$ and $P_{2}$ and tangent to $C$. Finally, there is a particular case when both $P_{0}$ and $P_{2}$ are inside the circle $C$ (Fig. 3r), in this case it should be stated than no annulus having $P_{0}$ and $P_{2}$ on one border and $P_{1}$ on the other can be found. 
a)

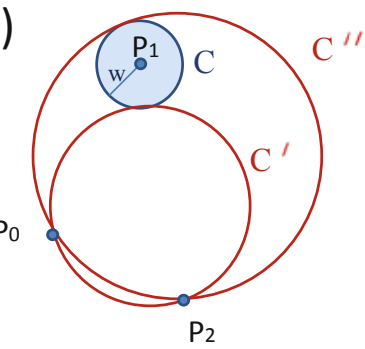

b)

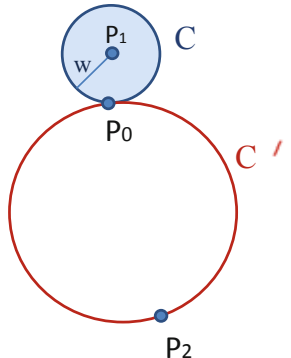

c)

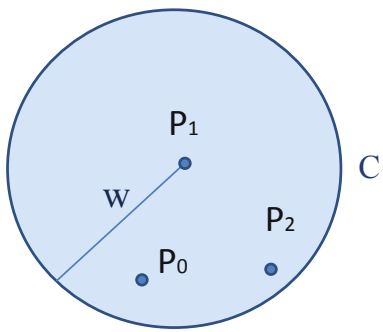

Fig. 3. Building of an annulus of width $\omega$ from 3 points: a) $P_{0}$ and $P_{2}$ are outside the circle $C$ of radius $\omega$ centered on $P_{1}$, b) $P_{0}$ is on the circle $C$, c) $P_{0}$ and $P_{2}$ are inside the circle $C$

\section{Finding the Optimal Fitting Annulus of Width $\omega$}

The principle of this naïve algorithm is to test all the possible triplets of points in $S$. For each triplet, we compute all the possible annuli (see theorem 2) and count the number of inliers. The optimal annulus (or the annuli) is the one that encloses the maximum number of inliers.

\subsection{Algorithm}

We now present Algorithm 1. Input is a set $S$ of $N$ discrete points and a thickness $w$ of our digital circle model. Output is a set $\mathbf{V}$ of parameter values $\left(C_{x}^{o p}, C_{y}^{o p}, R^{o p}\right)$ corresponding to the fitted annuli that give the optimal consensus sets. The time complexity of the algorithm is $O\left(N^{4}\right)$ because we have $N$ points in $S$ and every combination of three points defines each of the 8 circles, so taking the points three by three has a complexity of $O\left(N^{3}\right)$, then checking how many inliers and outliers we have for a given annulus is done in $O(N)$ time.

\subsection{Experiments}

We applied our method for 2D noisy digital Andres circles as shown in Fig. 4, 6, 7. For each of these set of points, an annulus of width $\omega=3, \omega=1$ and $\omega=1$ is used respectively. Table 1 shows the number of points, the optimal consensus set size as well as the center position and the radius $R$ of the inner circle obtained 


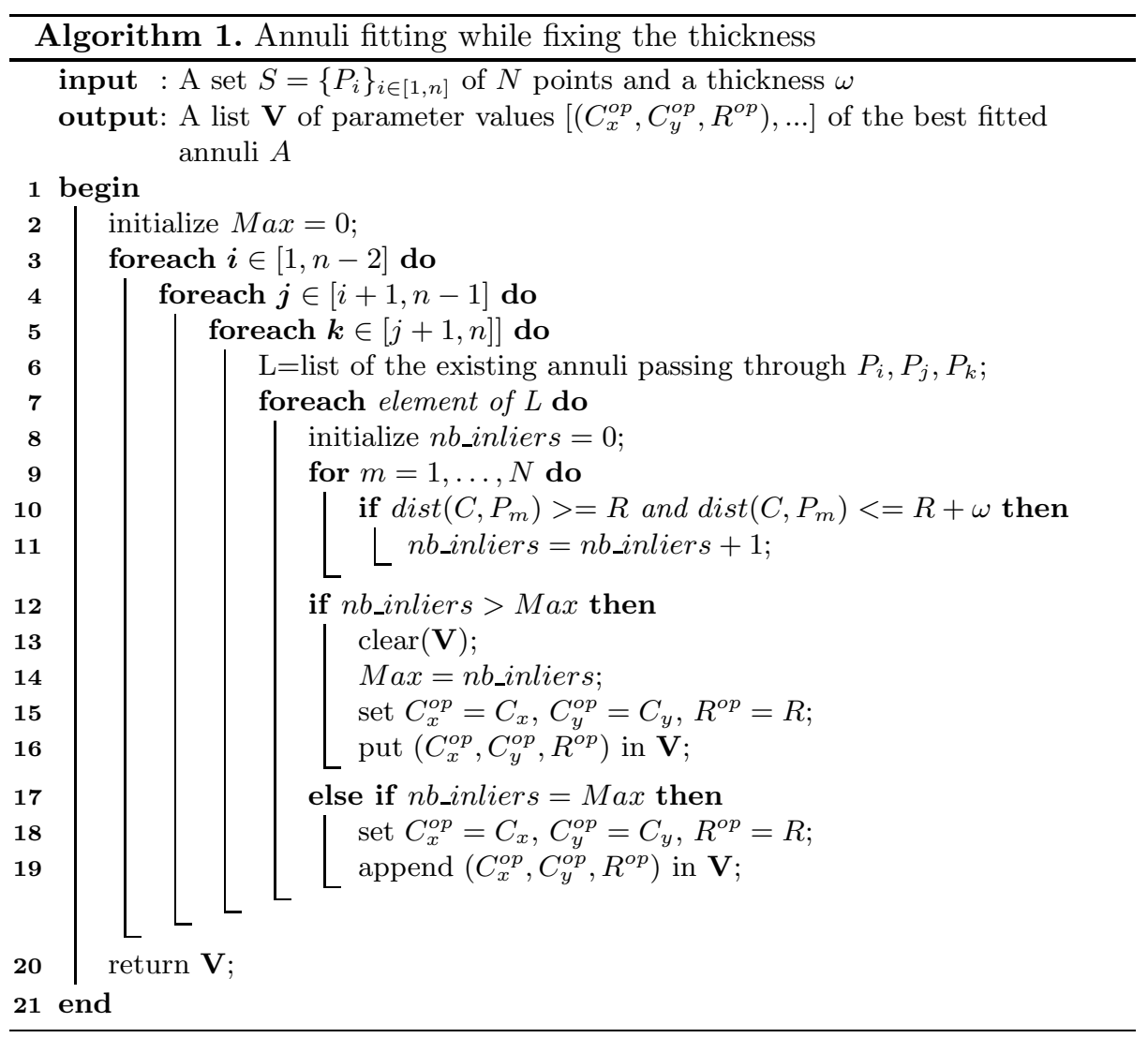

after the fitting. It should be noted that in Fig. 7, two optimal consensus sets are possible, since two annulus having the same number of inliers can be fitted (two circles in Fig. 7). This proves that our method is capable of detecting all optimal consensus sets. Our method is also applied to an Andres arc of width 1 as shown in Fig. 5, we can see that the arc of width $\omega=1$ is detected.

\section{Conclusion and Perspectives}

In this paper we have presented a new method for fitting annulus to a set of points while fixing the width of the annulus. Our approach is costly in terms of computation time however, its main advantage is that it guarantees optimal result from the point of view of maximal consensus set: we are guaranteed to fit an annulus with the least amount of outliers. This is the first time, to the author's best knowledge that and exact optimal methods dealing with the problem of annulus fitting with a fixed width with outliers has been proposed. One of the future work concerns the complexity of our approach. Our approach is rather brute-force and we obtain a $O\left(N^{4}\right)$ complexity. The question of improving this 
Table 1. The number of points and the optimal consensus set size for each of Fig. 4, 5. 6. 7

\begin{tabular}{|c|c|c|c|c|c|}
\hline Figures & Number of points & Center position & $\mathrm{R}$ & Thickness $w$ & Opt. consensus set size \\
\hline Fig. 4 & 289 & $(31,31)$ & 13 & 3 & 286 \\
Fig. 5] & 121 & $(101.581,102.226)$ & 86 & 1 & 118 \\
Fig. 6] & 119 & $(31,31)$ & 14 & 1 & 65 \\
Fig. 7 & 309 & $(31,31)(49,49)$ & 19 & 1 & 114 \\
\hline
\end{tabular}
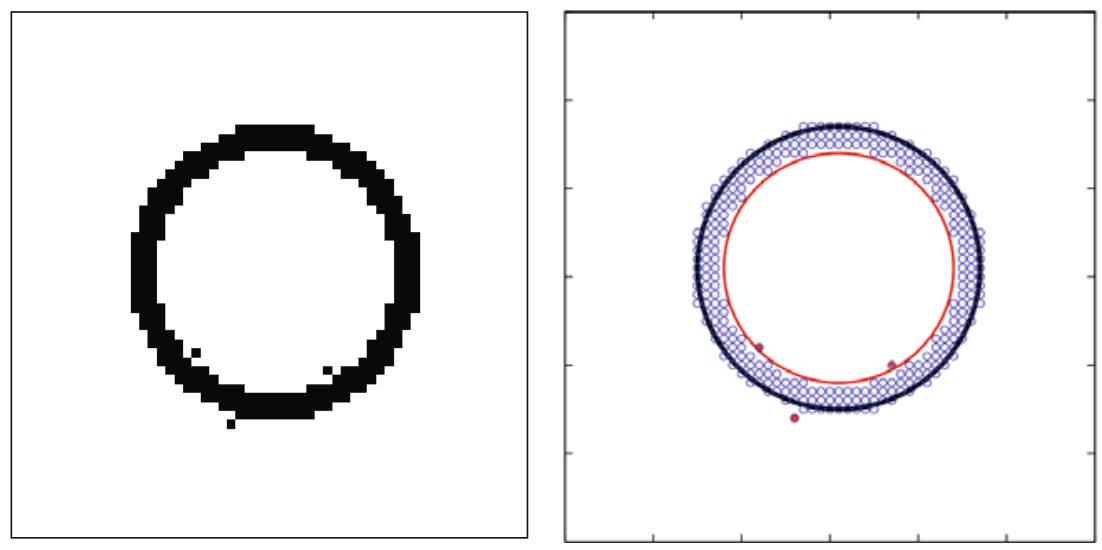

Fig. 4. Annulus fitting for a noisy digital Andres circle of width 3
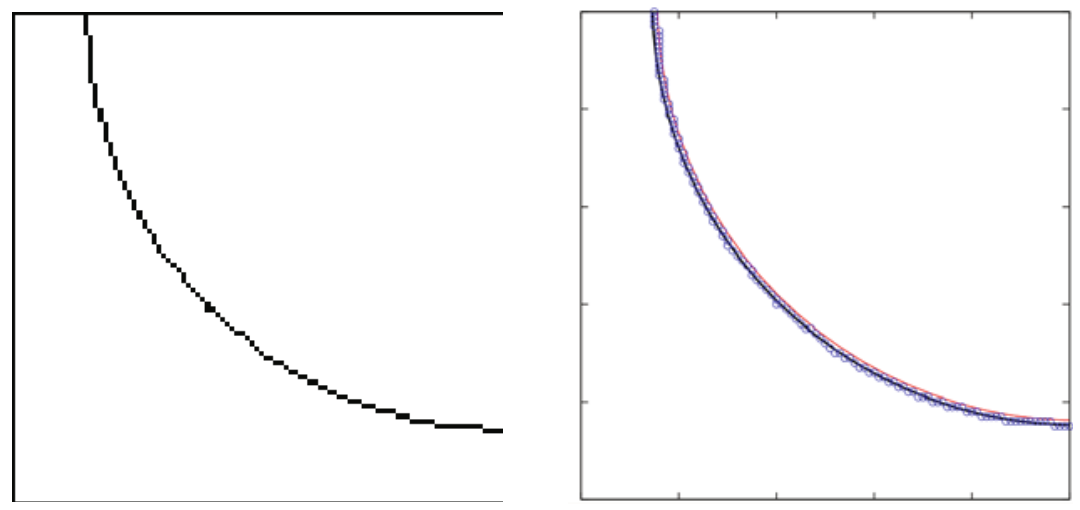

Fig. 5. Annulus fitting for a noisy digital Andres arc of width 1

complexity is open. We have wondered if this complexity may not be optimal. Indeed, it should be noted that fitting a digital plane (corresponding to two parallel continuous planes) to a given set of points in the presence of noise by maximizing the number of inliers is solved in $O\left(N^{3} \log N\right)$ [17. As we have 

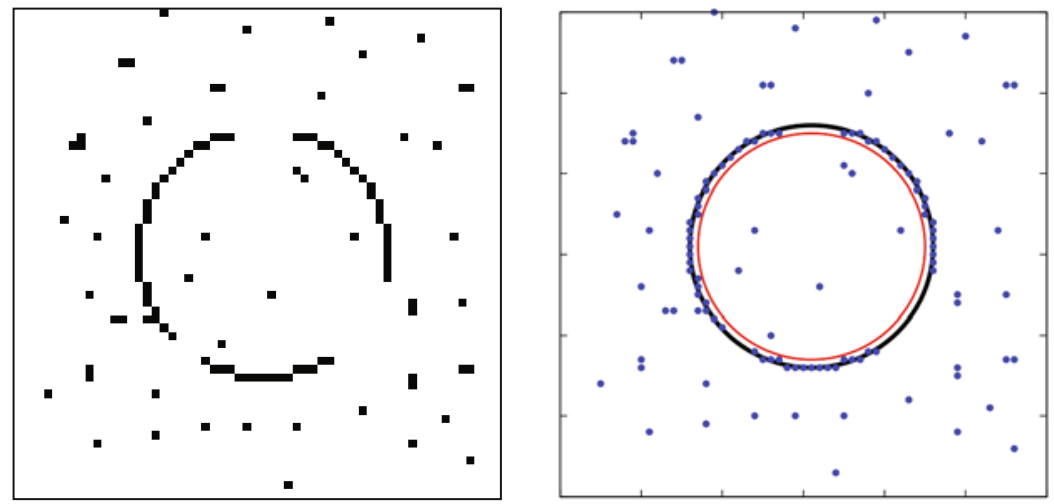

Fig. 6. Annulus fitting for a noisy image of a digital Andres circle of width 1
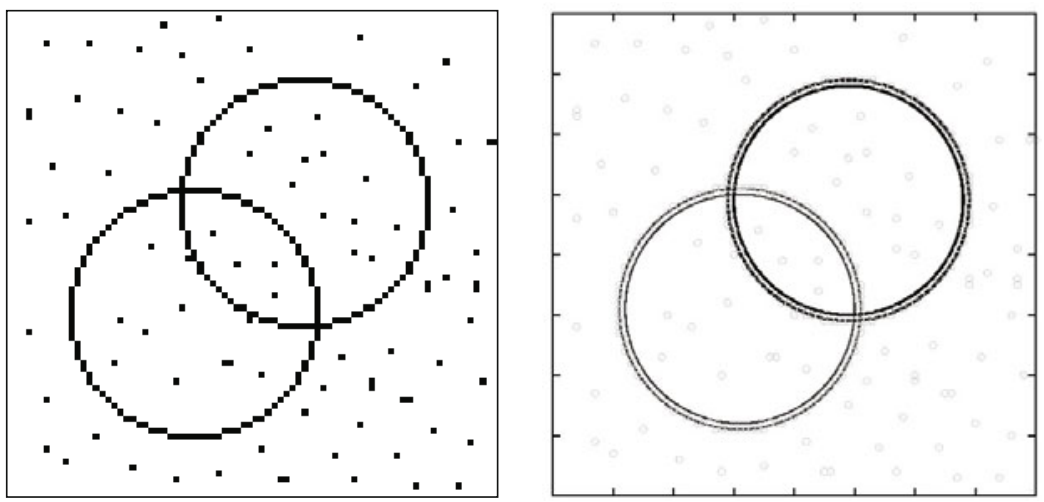

Fig. 7. Annulus fitting for two noisy digital Andres circle of width 1

explained in the introduction, there does not seem to be an immediate way of transforming our problem into a plane fitting problem with a O'Rourke type mapping [13. However, if such a way exists, we would still face a $O\left(N^{3} \log N\right)$ lower complexity limit. One planned extension is also the fitting of 3D annuli.

\section{References}

1. Agarwal, P.K., Har-Peled, S., Varadarajan, K.R.: Approximating extent measures of points. Journal of the ACM 51(4), 606-635 (2004)

2. Andres, E., Jacob, M.A.: The discrete analytical hyperspheres. IEEE Transactions on Visualization and Computer Graphics 3, 75-86 (1997)

3. Coeurjolly, D., Gérard, Y., Reveillès, J.P., Tougne, L.: An elementary algorithm for digital arc segmentation. Discrete Applied Mathematics 139(1-3), 31-50 (2004) 
4. Da Fontoura Da Costa, L., Cesar Jr., R.M.: Shape analysis and classification: Theory and practice, 1st edn. CRC Press, Inc., Boca Raton (2000)

5. De Berg, M., Bose, P., Bremner, D., Ramaswami, S., Ramaswami, G.: Computing constrained minimum-width annuli of point sets. In: Rau-Chaplin, A., Dehne, F., Sack, J.-R., Tamassia, R. (eds.) WADS 1997. LNCS, vol. 1272, pp. 392-401. Springer, Heidelberg (1997)

6. Ding, D., Ping, X., Hu, M., Wang, D.: Range image segmentation based on randomized hough transform. Pattern Recognition Letters 26(13), 2033-2041 (2005)

7. Dunagan, J., Vempala, S.: Optimal outlier removal in high-dimensional spaces. Journal of Computer and System Sciences 68(2), 335-373 (2004)

8. Dyer, M.E.: Linear time algorithms for two- and three-variable linear programs. SIAM Journal on Computing 13(1), 31-45 (1984)

9. Fischler, M.A., Bolles, R.C.: Random sample consensus: A paradigm for model fitting with applications to image analysis and automated cartography. Communications of the ACM 24, 381-395 (1981)

10. Har-Peled, S., Wang, Y.: Shape fitting with outliers. SIAM Journal on Computing 33(2), 269-285 (2004)

11. Matousek, J.: On enclosing $\mathrm{k}$ points by a circle. Information Processing Letters 53(4), 217-221 (1995)

12. Megiddo, N.: Linear-time algorithms for linear programming in $r^{3}$ and related problems. SIAM Journal on Computing 12(4), 759-776 (1983)

13. O'Rourke, J., Kosaraju, S.R., Megiddo, N.: Computing circular separability. Discrete and Computational Geometry 1, 105-113 (1986)

14. Rouche, E., de Comberousse, C., Poincare, H.: Traité de géométrie. Edition Jacques Gabay (1900), tomes I et II, 7e édition, Reprint (1997)

15. Roussillon, T., Sivignon, S., Tougne, L.: On three constrained versions of the digital circular arc recognition problem. In: Brlek, S., Reutenauer, C., Provençal, X. (eds.) DGCI 2009. LNCS, vol. 5810, pp. 34-45. Springer, Heidelberg (2009)

16. Smid, M., Janardan, R.: On the width and roundness of a set of points in the plane. International Journal of Computational Geometry 9(1), 97-108 (1999)

17. Zrour, R., Kenmochi, K., Talbot, H., Buzer, L., Hamam, Y., Shimizu, I., Sugimoto, A.: Optimal consensus set for digital plane fitting. In: Proceedings of 3DIM 2009 ICCV Satellite Workshop, pp. 1817-1824 (2009) 